\title{
MALASSEZIA PACHYDERMATIS E OUTROS AGENTES INFECCIOSOS NAS OTITES EXTERNAS E DERMATITES EM CÃES
}

\author{
MALASSEZIA PACHYDERMATIS AND OTHER INFECTIOUS AGENTS \\ IN EXTERNAL OTITIS AND DERMATITIS IN DOGS
}

\author{
Márcia Nobre $^{1}$ Mario Meireles $^{2}$ Luiz Fernando Gaspar ${ }^{1}$ Daniela Pereira $^{1}$ \\ Renata Schramm $^{3}$ Luiz Felipe Schuch ${ }^{1}$ Luciano Souza $^{4}$ Lorena Souza $^{4}$
}

RESUMO

Malassezia pachydermatis (Pityrosporum canis) faz parte da microbiota da pele e quando ocorrem alterações no microambiente local como aumento da umidade, da temperatura $e$ do substrato, determinando uma elevação do número de células, ocorre a transição da forma comensal para o parasitismo. $O$ objetivo deste trabalho foi determinar a prevalência da infeçâo por $\boldsymbol{M}$. pachydermatis e outros agentes infecciosos nas otites externas e dermatites de cães. Foram analisadas 78 amostras de cães com otite, 36 amostras de cães com o conduto auditivo normal e 24 com dermatite. Todas as amostras foram submetidas ao exame direto, cultivo em ágar sangue e em ágar Sabouraud dextrose, acrescido de cloranfenicol e cicloheximida. Ao exame direto, 50\% das amostras de otite e 16,6\% das amostras de dermatite apresentaram numerosas células semelhante a $\boldsymbol{M}$. pachydermatis, enquanto $83,3 \%$ das amostras de ouvidos saudáveis apresentaram ausência de células com esta morfologia. $\boldsymbol{M}$. pachydermatis foi isolada em $25 \%$ das amostras do conduto auditivo normal, em $80,7 \%$ das amostras de otite e em $37,5 \%$ das amostras de dermatite. Foram também isolados das amostras de otite Staphylococcus intermedius, S. aureus, S. epidermidis, Pseudomonas aeruginosa, Proteus sp, Actinomyces sp, Streptococcus sp e Otodectes cynotis e das de dermatite Staphylococcus intermedius, S. aureus, S. epidermidis Microsporum canis, $M$. gypseum,. Trychophyton sp e Demodex canis. M. pachydermatis foi o agente com maior prevalência nas otites externas e dermatites, sendo um agente oportunista que causa otite e dermatite quando há uma alteração do microambiente.

Palavras- chave: Malassezia pachydermatis, otites, dermatites, cães.

\section{SUMMARY}

The Malassezia pachydermatis ( Pityrosporum canis) is found in the whole skin microflora. It seems to occur as a consequence of changes in the local microenvironment, such as moist increase, temperature or substrate enhancement, generating a raising on cell quantity, which brings the transition from commensal form to parasitism. The purpose of this work was to evaluate the prevalence of $\boldsymbol{M}$. pachydermatis infection and other infectious agents in external otitis and dog dermatitis as well. We also examined 78 samples of dogs suffering from otitis, 36 samples of dogs having normal auditive tube and 24 having dermatitis. All samples were submitted to direct microscopic examination. They were cultured in blood agar, also in Sabouraud agar dextrose with cloranfenicol and cicloheximide. By dire't examination showed that $50 \%$ of the samples on dogs with otitis and dermatitis $16.6 \%$ showed several cels resembling $\boldsymbol{M}$. pachydermatis. However from healthy ear sample $83.3 \%$ did not present cells with this form. M. pachydermatis was isolated on $80.7 \%$ of otitis samples, on $25 \%$ of normal auditive tube samples and finally, $37.5 \%$ of dog dermatitis samples. It was isolated Staphylococcus intermedius, S. aureus, S. epidermidis, Pseudomonas aeruginosa, Proteus sp, Streptococcus sp and Otodectes cynotis from samples of animals with otitis. Cases of dermatites allowed also the isolation of Staphylococcus intermedius, $S$. aureus, S. epidermidis, Microsporum canis, M. gypseum, Trychophyton mentagophytes and Demodex canis. $M$. pachydermatis was the most prevalent agent in cases of external otitis and dermatitis, being an opportunistic agent, that cause otitis and dermatitis when there is a change in the microenvironment.

Key words: Malassezia pachydermatis, otitis, dermatitis, dog.

\footnotetext{
${ }^{1}$ Médico Veterinário, Professor da Faculdade de Veterinária da Universidade Federal de Pelotas (UFPel).

${ }^{2}$ Médico Veterinário, Doutor, Prof. Adjunto Depto. de Veterinária Preventiva, da Faculdade de Veterinária, UFPel, Campus Universitá rio s/n,96010-900, Pelotas, RS. E-mail: meireles@ufpel.tche.br. Autor para correspondência.

${ }^{3}$ Médico Veterinário, Faculdade de Veterinária, UFPel.

${ }^{4}$ Acadêmicos do Curso de Medicina Veterinária, Bolsistas de Iniciação Científica CNPq. Recebido para publicaçāo em 10.12.97. A provado em 01.04.98
} 


\section{INTRODUÇÃO}

Em 1925, WEIDMAN isolou de lesões de pele de rinoceronte indiano uma levedura lipofílicanão-dependente com gemulação unipolar em formato de "garrafa", sendo primeiramente denominada de Pityrosporum pachydermatis, posteriormente denominada Pityrosporum canis (GUSTAFSON, 1955) e atualmente é conhecida como Malassezia pachydermatis (SLOOFF, 1971; McGINNIS, 1980). O gênero Malassezia foi priorizado por ter sido descrito 15 anos antes (GORDON, 1980).

As leveduras do gênero Malassezia fazem parte da microbiota da pele Malassezia pachydermatis (Pityrosporum canis) que tem sido isolada tanto de cães sadios, com otite externa ou dermatite. Fatores que alteram o microclima local, fornecendo umidade, temperatura e substrato, estimulam o aumento do número de células desta levedura, fazendo-a passar da forma comensal ao parasitismo (FRASER, 1965; LARSON et al., 1988; LOBELL et al., 1995).

A $M$. pachydermatis se apresenta como células isoladas ou em grupo, medindo 2,0-2,5 x 4,0$5,0 \mu \mathrm{m}$, com formato oval ou com genulação unipolar, adquirindo o formato de "garrafa". As hifas e pseudo-hifas estão normalmente ausentes. O cultivo de materiais para isolamento da $\boldsymbol{M}$. pachydermatis é feito em ágar Sabouraud, a $25^{\circ} \mathrm{C}$ (durante 15 dias) ou a $37^{\circ} \mathrm{C}$ (durante 2 a 6 dias), acrescido de cloranfenicol e cicloheximida. O seu crescimento não é impedido pela ausência de lipídios, mas aumenta pela adição de azeite de oliva. As colônias são opacas, de coloração amarelo creme, passando a marrom alaranjado; a superfície é redonda ou em forma de cúpula; a textura é seca, friável e granulosa e algumas vezes gordurosa, medindo, após 5 dias de incubação a $32^{\circ} \mathrm{C}, 5 \mathrm{~mm}$ de diâmetro (MANSIFIELD et al., 1990; CUTSEM \& ROCHETTE; 1991; CORREA \& CORREA, 1992; QUINN et al., 1994; GUÉHO et al., 1996).

MANSIFIELD et al. (1990) realizaram um estudo experimental com 18 cães sadios, inoculando a $\boldsymbol{M}$. pachydermatis pura, em solução fisiológica ou em meio de cultura, no canal auditivo destes cães; após 21 dias do tratamento, todos apresentaram cultura positiva, sinais clínica e lesões histopatológicas, sendo o grupo controle $100 \%$ negativo. Os autores concluíram que $\boldsymbol{M}$. pachydermatis é um agente patogênico oportunista.

As otites externas e as dermatites apresentam causas multifatoriais, que provocam alteraçōes locais, seja de pH, umidade, temperatura, seja na microbiota, que facilitam o desenvolvimento de agentes secundários e oportunistas, dificultando o tratamento eficiente (MACY, 1992: LOBELL $\boldsymbol{e t}$ al., 1995; BOND et al., 1996). Tem sido relatado o isolamento da $\boldsymbol{M}$. pachydermatis e outros agentes infecciosos como Staphylococcus sp. , Pseudomonas sp., Proteus sp. e Streptococcus sp. de cães com otite externa ou dermatite por pesquisadores de diversos países (SZYNKIEWICZ et al., 1991; GENTILLINI et al., 1991; BORNAND, 1992; BOND et al., 1995;; KISS et al., 1997 ). No Brasil, os relatos de otites e dermatites por leveduras do gênero Malassezia são raros, com exceção dos trabalhos de FERNANDES \& GOMES, 1979; LARSSON et al., 1988; LANGONI et al., $1991 \mathrm{e}$ GIORGl et al., 1996. O presente trabalho foi desenvolvido com a finalidade de determinar a prevalência da $M$. pachydermatis e de outros agentes infeciosos em cães com conduto auditivo normal, com otites externas e com dermatites, com amostras provenientes de cinco municípios do Rio Grande do Sul (Jaguarão, Pelotas, Rio Grande, São Lourenço e Uruguaiana).

\section{MATERIAIS E MÉTODOS:}

No período de maio a dezembro de 1996 , foram analisadas no laboratório de Bacteriologia e Micologia da Faculdade de Veterinária da Universidade Federal de Pelotas (RS) 138 amostras de secreções de ouvido de cães, das quais, 36 procediam de cães sem otite, 78 de cães com otite e 24 de cães com dermatite. Foram considerados ouvidos normais aqueles sem presença de eritema, com cerumem normal e sem qualquer manifestação de dor. As coletas das amostras procedentes dos ouvidos foram feitas com "swabs", umedecidos em solução salina, friccionando-o na parte anterior do conduto auditivo externo. Nos cães com dermatite, foi feita a coleta através de raspado pele e/ou "swab". As amostras eram acompanhadas de dados clínicos dos cães fornecidos pelo clínico veterinário. Todas as amostras foram processadas no máximo em 24 horas após a coleta.

Para o exame direto da M. pachydermatis nas secreções de ouvido foram feitos esfregaços, utilizando a coloração pelo método de Gram. Nas escamas de pele foi utilizado uma fina camada de albumina de Meyer em uma lâmina de microscopia; sobre esta camada foram pressionados com o auxílio de agulha descartável, pequenos fragmentos das escamas no centro da lâmina e fixada ao calor. A lâmina foi corada pelo corante de Loeffler durante um minuto, deixando secar à temperatura ambiente, sendo então colocada uma lamínula sobre a lâmina, fixando com bálsamo do Canadá. Ambas preparações foram observadas ao microscópio (1000X) em 
busca de células semelhantes a $\boldsymbol{M}$. $\boldsymbol{p} \boldsymbol{a}$ chydermatis. O exame direto foi avaliado, contando as células morfologicamente semelhantes a $\boldsymbol{M}$. pachydermatis/campo, considerando o seguinte escore: (-) ausência de células compatíveis /campo; (+) até 5 células/campo; (++) 5 a 10 células/campo e (+++) mais de 10 células/campo. O exame direto com $\mathrm{KOH}$ a $20 \%$ ou glicerina entre lâmina e lamínula foi utilizado para o diagnóstico, respectivamente para dermatófitos e sarnas e visualizado em microscopia ótica(X400).

O material coletado foi cultivado em placas de Petry, contendo ágar Sabouraud dextrose, acrescido de cloranfenicol e cicloheximida, para avaliação das características macroscópicas e microscópicas de leveduras e dermatófitos, mantidos à $37^{\circ} \mathrm{C}$ por até 10 dias. As colônias obtidas após a incubação foram identificadas bioquimicamente segundo LACAZ et al., 1984 e DWORECKAKASZAK et al., 1994. O material também foi cultivado em ágar sangue, $8 \%$ de sangue desfibrinado de ovino, mantido a $37^{\circ} \mathrm{C}$ durante 48 horas, para o isolamento de bactérias, que foram identificadas segundo QUINN et al. (1994).

\section{RESULTADOS E DISCUSSÃO}

No exame direto das amostras dos ouvidos clinicamente normais, 83,3\% não apresentaram células morfologicamente compatíveis com $\boldsymbol{M}$. $\mathbf{p a}$ chydermatis e nenhuma amostra apresentou mais de 10 células/campo. Das amostras provenientes de otites, $50 \%$ apresentaram mais de 10 células/campo e em somente $12,8 \%$ não foram encontradas células de $\boldsymbol{M}$. pachydermatis. O exame direto das amostras procedentes de dermatite revelou mais de 5 células/campo em $33,2 \%$, enquanto $62,5 \%$ apresentaram ausência da levedura (Tabela 1).

Nas 138 amostras estudadas, o maior índice de isolamento da $\boldsymbol{M}$. pachydermatis foi nas otites $(80,7 \%)$, seguidas das dermatites $(37,5 \%)$, enquanto os ouvidos normais apresentaram $25 \%$ (Tabela 2), sendo isolada como único agente em $21,7 \%$ das amostras de otite. Das amostras dos cães sem otite, o único agente infeccioso isolado foi a $\boldsymbol{M}$. pachydermatis e das amostras de cães com otite externa foram também isolados agentes bacterianos (Staphylococcus aureus, Staphylococcus intermedius, Staphylococcus epidermidis, Streptococcus sp, Actinomyces sp, Pseudomonas aeruginosa e Proteus sp) e parasitários (Otodectes cynotis). Nas der-
Tabela 2 - Freqüência de isolamento de M. pachydermatis em 138 amostras de cães com ouvidos normais, com otite externa e com dermatite.

\begin{tabular}{cccc}
\hline Natureza das & Positivos & Negativos & Total \\
amostras & $\mathrm{N}^{\circ}(\%)$ & $\mathrm{N}^{\circ}(\%)$ & $\mathrm{N}^{\circ}(\%)$ \\
Sem otite & $9(25,0)$ & $27(75,0)$ & $36(26,1)$ \\
Com otite & $63(80,7)$ & $15(19,2)$ & $78(56,5)$ \\
Com dermatite & $9(37,5)$ & $15(62,5)$ & $24(17,4)$ \\
Total & $81(58,7)$ & $57(41,3)$ & $138(100)$
\end{tabular}

$\mathrm{N}^{\circ}=$ Freqüência absoluta; $(\%)=$ Freqüência relativa .

matites, além de terem sido isolados Staphylococcus aureus, Staphylococcus intermedius e Staphylococcus epidermidis também foram encontrados Trichophyton mentagrophytes, Microsporum canis, Microsporum gypseum e Demodex canis (Tabela 3). Das 78 amostras de otites, 4\% (3) não apresentaram parasitas, nem crescimento de bactérias ou fungos. O mesmo ocorreu em $8 \%$ (2) das amostras de cães com dermatite. No cadastro destes animais constava a informação de que haviam interrompido o tratamento com antibióticos há pouco tempo ou ainda estavam sendo tratados.

$\mathrm{O}$ agente isolado com maior frequiência dos cães com dermatite foi $\boldsymbol{M}$. pachydermatis $(37,5 \%)$, estando sempre associada com outros microrganismos, principalmente $S$. intermedius (55,6\%) e Demodex canis $(22,2 \%)$ e em menor freqüência com S. aureus (11,1\%), e Trichophyton mentagrophytes $(11,1 \%)$ (Tabela 4). Em todos os casos nos quais a $\boldsymbol{M}$. pachydermatis foi isolada, a dermatite era crônica, generalizada e com intenso prurido. Nas amostras em que estava associada com 


\begin{tabular}{|c|c|c|c|}
\hline \multirow[b]{2}{*}{ Agentes } & \multicolumn{3}{|c|}{ Natureza das amostras } \\
\hline & $\begin{array}{l}\text { Sem otite } \\
\mathrm{N}^{\circ}(\%)\end{array}$ & $\begin{array}{c}\text { Com otite } \\
\mathrm{N}^{\circ}(\%)\end{array}$ & $\begin{array}{c}\text { Com dermatite } \\
\mathrm{N}^{\circ}(\%)\end{array}$ \\
\hline M. pachydermatis & $9(25)$ & $63(80,7)$ & $9(37,5)$ \\
\hline S. intermedius & ND & $16(20,5)$ & $7(29,16)$ \\
\hline S. aureus & ND & $12(15,4)$ & $6(25)$ \\
\hline P. aeruginosa & ND & $8(10,3)$ & ND \\
\hline Actinomyces sp & ND & $8(10,3)$ & ND \\
\hline Proteus sp & ND & $7(8,9)$ & ND \\
\hline S. epidermidis & ND & $5(6,4)$ & $2(8,3)$ \\
\hline Streptococcus sp & ND & $3(3,8)$ & ND \\
\hline M. canis & ND & ND & $1(4,1)$ \\
\hline M. gypseum & ND & ND & $1(4,1)$ \\
\hline T. mentagrophytes & ND & ND & $2(8,3)$ \\
\hline Demodex canis & ND & ND & $3(12,5)$ \\
\hline Otodectes cynotis & ND & $1(1,2)$ & ND \\
\hline
\end{tabular}

ND = Não detectado; $N^{\circ}=$ Freqüência absoluta; $(\%)$ = Freqüência relativa.

Demodex canis, os casos atuais eram de recidiva e nos casos em que estava associada ao $\boldsymbol{S}$. intermedius havia suspeita de processo alérgico.

Este estudo demonstra que entre todos os agentes isolados de cães com otite, a M. pachydermatis foi o mais freqüente com uma prevalência de $80,7 \%$; resultados similares aos encontrados por SMITH (1968), que obteve uma prevalência de 95,5\% e os de SZYNKIEWICZ et al. (1991) de $71,2 \%$, enquanto BORNAND (1992) descreveu a $\boldsymbol{M}$. pachydermatis com uma prevalência de $56 \%$. Os nossos resultados são discordantes daqueles obtidos por GIORGI et al. (1996) que isolaram a $\boldsymbol{M}$. pachydermatis em 10,2\% de cães clinicamente com otite e de GENTILLINI $\boldsymbol{e t}$ al. (1991), que ao investigarem a flora micótica e bacteriana de 91 amostras de otites crônicas, encontraram uma prevalência de $7,7 \%$. Cabe ressaltar, entretanto, que nas 78 amostras em estudo foi isolado $10,3 \%$ de $\boldsymbol{P}$. aeruginosa, sem isolamento de $\boldsymbol{M}$. pachydermatis (Tabela 4), enquanto GENTILLINI et al. (1991) encontraram uma prevalência de $51,6 \%$ de $\boldsymbol{P}$. aeruginosa, sendo que destes houve uma associação entre os dois agentes.

A prevalência da $\boldsymbol{M}$. pachydermatis em cães saudáveis foi de $25 \%$, o que é compatível com os $22 \%$ encontrados por BORNAND (1992), que representam a microbiota fúngica normal, porém frontalmente discordante dos resultados de NUNES \& HAMDAN (1995) que encontraram a levedura em
91,4\% das amostras de cães sem otite. Nos cães sem otite, considerados com ouvidos normais, a M. pachydermatis foi o único agente isolado, provavelmente devido à seleção dos ouvidos, que apresentavam um conduto auditivo sem eritema e sem excesso de cerumem, enquanto BORNAND (1992), além da M. pachydermatis, também isolou $11 \%$ de $S$. intermedius.

\section{As bactérias $S$. intermedius,} S.aureus, P. aeruginosa, Actinomyces sp, Proteus $s p, S$. epidermides e Streptococcus $s p$, isoladas de amostras de secreção de ouvido de cães com otite, foram as mesmas encontradas pela maioria dos autores (SMITH, 1968; GENTILLINI et al., 1991, SZYNKIEWICZ et al., 1991; BORNAND, 1992; GIORGI et al., 1996). Quanto à prevalência dos agentes bacterianos, de $15,4 \%$ para o $S$. aureus, de $20,5 \%$ para o $\boldsymbol{S}$. intermedius e de $10,3 \%$ para o $\boldsymbol{P}$. aeruginosa., diferem das freqüências encontradas por SMITH (1968) que obteve uma prevalência de 36,4\% de S. aureus; SZYNKIEWICZ et al.. (1991) que encontraram $S$. intermedius em $51 \%$ das amostras e GENTILLINI et al. (1991) isolaram $\boldsymbol{P}$. aeruginosa em 51,6\%, $\boldsymbol{S}$. epidermidis em 5,5\% e $S$. aureus em 1,1\%.

M. pachydermatis foi isolada em $37,5 \%$ das amostras de dermatite. Clinicamente, estas dermatites se caracterizavam como crônicas, generalizadas e pruriginosas, resultados estes concordantes com aqueles observados pela maioria dos autores (DUFTAI, 1983; LARSON et al., 1988; SCOTT \& MILLER, 1989; STEWART, 1990; BOND et al., 1995; BOND et al.,1996). Nesses cães, independente da causa primária (processo alérgico, distúrbios de queratinização, presença de parasitas) havia um aumento da umidade e da temperatura da epiderme, que é adequado ao desenvolvimento de agentes oportunistas como a $\boldsymbol{M}$. pachydermatis. O segundo microrganismo mais freqüente foi o $S$. intermedius com $29,2 \%$, muito aquém dos resultados encontrados por SZYNKIEWICZ et al. (1991) que observaram uma freqüência de $70,3 \%$, e destes $14,6 \%$ estavam associados a $M$. pachydermatis. Nos resultados obtidos, houve 9 isolamentos de $\boldsymbol{M}$. $\mathbf{p a}$ chydermatis (TABELA 4), dos quais em dois havia a presença do Demodex canis, semelhante aos resultados de LARSSON et al.(1988), que diagnosticaram 23 casos de dermatites causadas por $\boldsymbol{M}$. pachydermatis, com a presença do Demodex canis em 3 casos.

A freqüência da $M$. pachydermatis no sul do Brasil foi maior em otites (63) do que em dermatites (9), enquanto ALMEIDA et al. (1995), na região sudeste encontraram uma maior freqüência da 


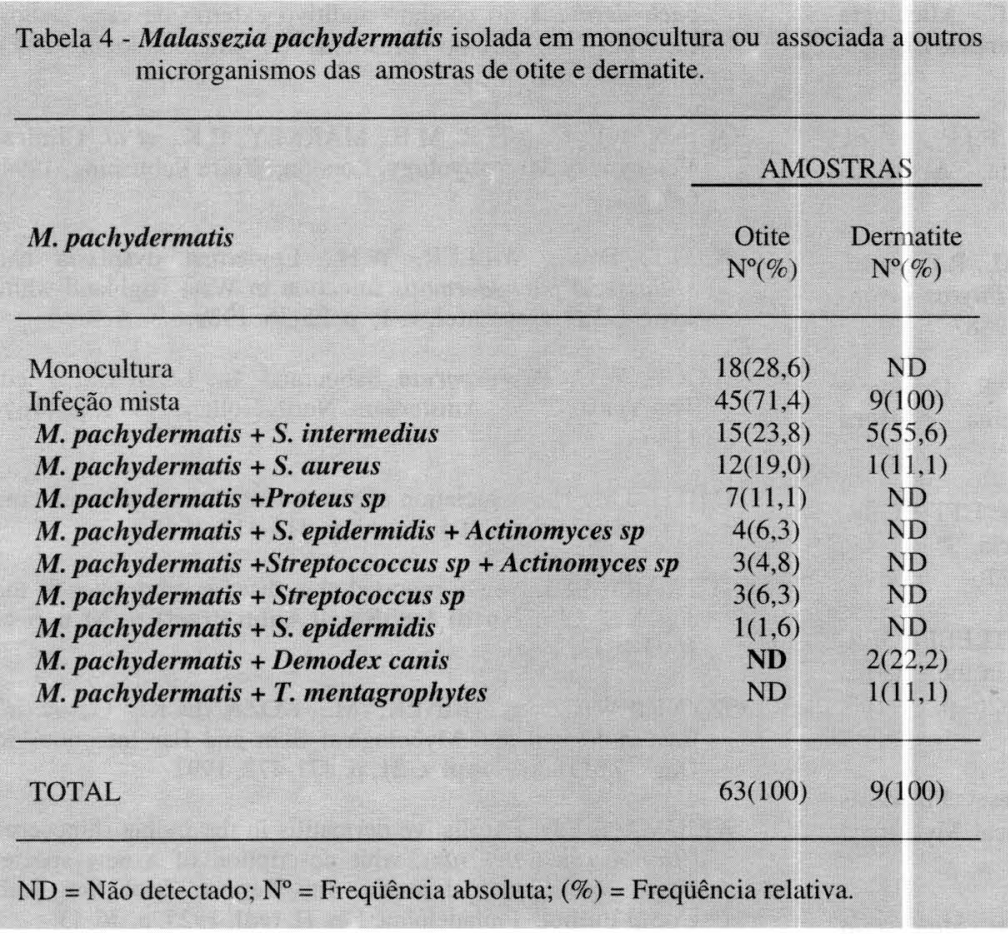

levedura em dermatites (30), seguido de otites (19), tendo também encontrado um caso de onicomicose. Os resultados obtidos permitem concluir que a $\boldsymbol{M}$. pachydermatis foi o agente com maior prevalência nas otites, atuando associada ou isoladamente, enquanto nas dermatites se apresenta sempre associada a outros agentes. Havendo um desequilíbrio do microambiente, há uma tendência ao aumento do número de células de $\boldsymbol{M}$. pachydermatis, sendo o exame direto, através da contagem de células, um método efetivo para avaliar a forma de parasitismo.

O diagnóstico laboratorial e a identificação dos agentes nestas enfermidades, seguidos de testes de sensibilidade aos antimicrobianos se fazem necessários para evitar a seleção de cepas resistentes e a alteração na microbiota, o que facilitaria o desenvolvimento de agentes oportunistas, como a $\boldsymbol{M}$. pachydermatis.

\section{AGRADECIMENTOS}

Os autores agradecem ao professor Claudiomar Soares Brod pelo auxílio na fase de análise epidemiológica e ao apoio financeiro do $\mathrm{CNPq}$ (Bolsa Iniciação Científica) e da CAPES (Bolsa de Mestrado). Também somos gratos às Clínicas Veterinárias da região pelo envio dos casos.

\section{REFERÊNCIAS BIBLIOGRÁFICAS}

ALMEIDA, R.M.A., PARRA, T., DEL BIANCHI, M. Malassezia pachydermatis - Agente de dermatites, otites e onicomicose em cães: Diagnóstico clínico e micológico. In: XVIII Congresso Brasileiro de Microbiologia, 1995. Santos,
SP, Anais: p. 133.

BOND, R., ROSE, J.F., ELLIS, J.W., et al. Comparison of two shampoos for treatment of Malassezia pachydermatis associated seborrhoeic dermatitis in basset hounds. J Small Anim Pract, v. 36, p. 99-104, 1995.

BOND, R., FERGUSON, E.A., CURTIS, C.F., et al. Factors associated whit elevated cutaneous Malassezia pachydermatis population in dogs whit pruritic skin disease. J Small Anim Pract, v. 37, p. 103-107, 1996.

BORNAND, V. Bactériologie et mycologie de l'otite externe du chien. Schweiz Arch Tierheilk, v. 134, p. 1-8, 1992.

CORRÊA,W.M., CORRÊA, C.N.M. Enfermidades dos mamíferos domésticos. 2. ed, Rio de Janeiro, Editora Médica e Científica, 1992, $843 \mathrm{p}$

CUSTSEM, V.J., ROCHETTE, F. Mycosis in Domestic Animals. Janssen Research Foundation, 1991, 226p.

DOWORECKA-KASZAK, B., SYNKIEWICZ, Z., BLASZCZAK, B. - Evaluation of selected physiological and morphological characteristics of Pityrosporum pachydermatis isolated from clinical cases of Otitis externa and Dermatitis in dogs and cats. Arch Vet Polonicum, v. 3, p. 3-

4, 1994

DUFAIT, R. Pityrosporum canis as the cause of canne chronic dermatitis. Vet Med (Small Anim Clinc) p. 1055-1057, 1983.

FERNANDES, J.C.T., GOMES, M. Flora bacteriana e fúngica de cães com otite atendidos pelo Hospital de Clínicas Veterinárias da UFRGS. Arq Fac Vet UFRGS, Porto Alegre v. 7, p. 97-103, 1979.

FRASER, G. A etiology of otitis externa in the dog. J Small Anim Pract, v. 6, p. 445, 1965.

GENTILLINI, E., DENAMIEL, G.A.A., ESCALADA, J., $\boldsymbol{e}$ al. Otitis canina cronica hallazgos microbiologicos y sensibilidad a los antibioticos. Vet Arg v. 8, n. 72, p. 113-117, 1991

GIORGI, W., MARTIN, C.M., SCHMIDT, E.F. Principais agentes etiológicas da otite externa em cães. Pet Vet, v. 2, p. $15-17,1996$.

GUÉHO, E., MIDGLEY, G., GUILlOT, J. The genus Malassezia with description of four new species. Antonie van Leeuwenhoek, v. 69, p. 337-355, 1996.

GORDON, M.A. How superficial is Malassezia furfur? In: THE FIFTH INTERNATIONAL CONFERENCE ON THE MYCOSES. 1980, Caracas. Proceedings.... Pan American Healt-Organization, 1980 , p. $38-43$, (Scientific Publication $n^{\circ}$ 396).

GUSTAFSON, B.A. Otitis externa in the dog: a bacteriological and experimental study. Royal Veterinary College of Sweden, Stockholm, 1955.

KISS G., RADVÁNYI, Sz., SZIGETI, G., et al. New combination for the therapy of canine otitis externa II Eficacy in vitro and in vivo. J Small Anim Pract, v. 38, p. 57-60, 1997. 
LACAZ, C.S., PORTO E., MARTINS, J.E.C. Micologia Médica: fungos, actinomicetos e algas de interesse médico. $7^{\mathrm{a}}$ ed., São Paulo, Sarvier, 1984, 479 p.

LANGONI, H., FESSEL, Y.M.N., LISTONI, F.J.P., $\boldsymbol{e t}$ al. Microflora aeróbica de ouvido de cães sem otite. Arq Bras Med Zootec, v. 43, n. 3, p. 255-260, 1991.

LARSSON, C.E., LARSON, M.H.M.A., AMARAL, R.C., et al. Dermatitis in dogs caused by Mallassezia (Pityrosporum) pachydermatis. Ars Vet, v. 4, n.1, p. 63-68, 1988.

LOBELL, R., WEINGARTEN, A., SIMMONS, R. Um novo agente para o tratamento da otite externa canina. A Hora Veterinária, v. 88, p. 29-33, 1995.

MACY, D.W. Moléstias do aparelho auditivo. In: ETTINGER, S.J, Tratado de Medicina Interna Veterinária, $3^{\mathbf{a}}$ ed, São Paulo, Editora Manole Ltda, 1992, v. 1, p. 256-276

MANSFIELD, P.D., BOOSINGER, T.R., ATTLEBERGER, M.H. Infectivity of Malassezia pachydermatis in the external ear canal of dogs. J Am Anim Hosp Ass, v. 26, p. 97-100, 1990

McGINNIS, R.M. Sinopsis of the mycoses: Pityriasis versicolor. In: Laboratory Handbook of Medical Mycology, London. Academic Press, 1980, p. 475-522.

NUNES, B.M., HAMDAN, J.S. Prevalência de Malassezia pachydermatis no conduto auditivo externo de cães sadios. In: XVIII Congresso Brasileiro de Microbiologia, 1995. Santos, SP., Anais: p. 132

QUINN, P.J., CARTER, M.E., MARKEY, B.K., et al. Clinical Veterynary Microbyology. London, Wolfe Publishing, 1994, $648 \mathrm{p}$.

SCOTT, D.W., MILLER, W.H., Epidermal dysplasia and Malassezia pachydermatis infection in West Highland white terriers. Vet Dermatol, v. 1, p. 25-36, 1989.

SLOOF, W.C. Pityrosporum Sabouraud. In: LODDER, J. ed. The Yeasts $2^{a}$ ed., Amsterdam, North-Holland, 1971, p. 1167 1186.

SMITH, J.M. The association of yeast whit chronic otitis externa in the dog Aust Vet J, v. 44, p. 413-415, 1968.

STEWART, L. J. Newly reported skin disease syndromes in the dog. Vet Clin North Am (Small Anim Pract), v. 20, n. 6, p. $1603-1613,1990$.

SZYNKIEWICZ, Z., BINEK, M., KOZANECKI, C., et al. Bacteriological and Mycological Skin and Ear Infections in Dogs Zbl Bakt Suppl v. 21, p. 471-472, 1991.

WEIDMAN, F.D. Exfoliative dermatitis in the Indian rhinoceros (Rhinoceros unicornis), whit description of a new specie: Pityrosporum pachydermatis. In: Report Zool Soc Lab Comp Pathol, Philadelphia, Fox H. (ed), 1925, p. 36-43.

Ciência Rural, v. 28, n. 3, 1998. 